Documentation et bibliothèques

DOCUMENTATION BIBLIOTHEQUES

\title{
Les évolutions technologiques récentes et le milieu documentaire
}

\section{Recent technological advances in libraries}

\section{Las evoluciones technológicas recientes y el medio documental}

\section{Denis Varloot}

Volume 32, numéro 1-2, janvier-juin 1986

URI : https://id.erudit.org/iderudit/1052709ar

DOI : https://doi.org/10.7202/1052709ar

Aller au sommaire du numéro

Éditeur(s)

Association pour l'avancement des sciences et des techniques de la documentation (ASTED)

ISSN

0315-2340 (imprimé)

2291-8949 (numérique)

Découvrir la revue

Citer cet article

Varloot, D. (1986). Les évolutions technologiques récentes et le milieu documentaire. Documentation et bibliothèques, 32(1-2), 29-32.

https://doi.org/10.7202/1052709ar
Résumé de l'article

L'auteur décrit les modifications que les évolutions technologiques récentes ont entraînées dans le milieu documentaire universitaire en France. Après avoir recensé brièvement les innovations technologiques intervenues dans le domaine de la documentation, il montre comment les nouvelles technologies modifient les équilibres existant entre les différents acteurs du réseau documentaire. Il expose enfin quelques-uns des problèmes que ces mutations entraînent chez les professionnels de la documentation.
Tous droits réservés (c) Association pour l'avancement des sciences et des techniques de la documentation (ASTED), 1986
Ce document est protégé par la loi sur le droit d'auteur. L’utilisation des services d’Érudit (y compris la reproduction) est assujettie à sa politique d'utilisation que vous pouvez consulter en ligne. 


\title{
Les évolutions technologiques récentes et le milieu documentaire
}

\author{
Denis Varloot* \\ Ministère de l'Éducation nationale \\ Paris
}

L'auteur décrit les modifications que les évolutions technologiques récentes ont entraînées dans le milieu documentaire universitaire en France. Après avoir recensé brièvement les innovations technologiques intervenues dans le domaine de la documentation, il montre comment les nouvelles technologies modifient les équilibres existant entre les différents acteurs du réseau documentaire. II expose enfin quelques-uns des problèmes que ces mutations entraînent chez les professionnels de la documentation.

\section{Recent technological advances in libraries}

The author describes recent technological changes in French university libraries. Following a brief review of the technological changes in libraries, he outlines how new technologies modify the equilibrium among the various professionals of the library network. Finally, he identifies some of the problems these changes bring to information professionals.

\section{Las evoluciones technológicas recientes y el medio documental}

El autor describe las modificaciones que trajeron las evoluciones tecnológicas recientes al medio documental universitario en Francia. Después de enumerar brevemente las innovaciones tecnológicas que intervinieron en el campo de la documentacion, demuestra como las nuevas tecnologias modifican los equilibrios existentes entre los diferentes actores de la red documental. Por fin, expone algunos de los problemas que estos cambios plantean entre los profesionales de la documentación.
Si déterminantes qui puissent être certaines innovations technologiques, leur impact est conditionné par les facteurs humains, par la capacité du groupe social à intégrer les innovations, à en susciter, à en bloquer ou en rejeter. Or, nous devons être tout à fait conscients que les générations actuelles vivent des mutations technologiques sans commune mesure avec celles qu'ont vécues nos ancêtres. En effet, depuis une quinzaine d'années, nous sommes passés d'une ère où I'on rencontrait une révolution technologique toutes les deux ou trois générations à une ère dans laquelle nous sommes encore, où chaque génération doit s'adapter à une ou plusieurs révolutions technologiques.

\section{Les nouvelles technologies ont bouleversé le} paysage documentaire

Pour la France, mentionnons les innovations suivantes:
- au niveau des utilisateurs d'information

- la création d'un réseau de transmission de données à faible coût (Transpac);

- I'explosion du vidéotex: un million et demi de Minitel en France;

- la baisse du coût des postes de travail permettant de traiter en local des volumes importants d'information (micro-ordinateurs et lecteurs de disques compacts).

- au niveau de l'exploitation et de la diffusion de l'information

- l'évolution des logiciels de diffusion de l'information;

- le développement des interfaces (user friendly systems) en réponse au tassement de la consommation sur l'ensemble des grands serveurs qui s'adressent à un public d'intermédiaires; 
- le développement des services locaux sur micro-ordinateurs dont la capacité de mémoire s'est accrue grâce au disque optique numérique, et qui peuvent désormais traiter des fonds importants.

- au niveau de la production d'information

- le développement d'outils de messagerie;

- l'indexation assistée par ordinateur;

- I'utilisation de la lecture optique pour la saisie des données;

- la traduction assistée par ordinateur.

\section{Les nouvelles technologies modifient les équilibres entre les différents groupes participant au réseau documentaire}

Ces innovations n'interviennent pas dans un contexte social neutre, mais dans la société française contemporaine. II peut être utile de rappeler certaines caractéristiques de cette société car elles doivent être prises en compte pour l'introduction des nouvelles technologies dans le milieu documentaire:

- un processus de décentralisation est en cours;

- le réseau documentaire est un milieu faiblement structuré;

- des secteurs entiers sont fortement cloisonnés, comme le secteur scolaire ou le secteur associatif.

Par ailleurs, les groupes concernés appartiennent à des secteurs variés: professionnels de l'information, administratifs et techniciens, usagers, représentants des administrations centrales, organisations syndicales, associations ..., mais aussi collectivités locales, universités et établissements d'enseignement se réappropriant leur documentation.

Les équilibres existant entre ces divers groupes se trouvent modifiés par l'introduction des nouvelles technologies, en particulier l'équilibre décideurs-utilisateurs-acteurs.

- Les décideurs

L'implantation des équipements n'est pas régie par le marché, mais résulte d'arbitrages entre politique documentaire et politique industrielle, marqués par les contraintes internes telles que les restrictions budgétaires, et externes, au premier rang desquelles la place dans l'économie mondiale.

Comment faire évoluer le système de décision pour avoir les meilleures conditions d'implantation?
- Les utilisateurs

Les nouvelles technologies imposent souvent une collaboration entre les institutions. Ainsi, pour la mise en place d'équipements lourds, la charge des investissements est assurée conjointement par plusieurs instances: administrations centrales (Recherche, Éducation, Culture), collectivités territoriales (région, commune)...

Ce mode de collaboration est nouveau; il occasionne de multiples interrogations, telles que: quels sont les supports institutionnels qui existent pour ces mises en contact? Quel mode d'introduction des nouvelles techniques doit être adopté selon qu'il s'agit d'une décision centralisée ou d'une initiative locale? Quelles sont les solutions les plus prometteuses sur le plan humain?

\section{- Les acteurs}

Les nouvelles technologies font entrer en scène de nouveaux acteurs: les ingénieurs spécialistes et les sociétés de services; par ailleurs, elles redonnent aux utilisateurs finals un rôle déterminant.

Comment va se caractériser la nouvelle donnée? Comment se répartiront les tâches et les fonctions entre les divers acteurs?

Pour les usagers en particulier, l'introduction des nouvelles technologies est l'occasion d'une nouvelle approche de l'information: il leur faut apprendre à chercher et à évaluer l'information; aussi est-il primordial qu'ils se familiarisent très tôt avec les nouvelles technologies et acquièrent des réflexes de base qui rendront possible l'actualisation ultérieure. À cet effet, de nombreuses actions de sensibilisation à l'utilisation de l'information scientifique et technique sont menées en milieu universitaire: introduction dans le cursus des études d'un enseignement pratique des sciences et techniques de la documentation; formation à l'interrogation des banques de données; financement partiel des interrogations de banques de données françaises (opération Ticket Modérateur); télématique universitaire mettant à la disposition de la communauté des services tels que journal cyclique, annuaire, messagerie ...; plan Informatique Pour Tous visant à initier à l'informatique les élèves de l'ensemble des établissements publics à tous les niveaux d'enseignement ...

\section{Les nouvelles technologies transforment l'exercice des professions de la documentation}

Petit à petit l'introduction des nouvelles tech- 
nologies s'étend à toutes les tâches, toutes les fonctions exercées dans les bibliothèques et centres de documentation pour accueillir, orienter et conseiller les usagers, puis pour satisfaire leurs besoins de lecture ou d'information: recherche bibliographique et documentaire, consultation sur place de documents sous des formes de plus en plus variées (disques, microformes, films, diapositives, cassettes, son vidéo, vidéodisques, logiciels ...) prêt à domicile (local ou interbibliothèque), fourniture de documents à distance (télécopie, transmission électronique de documents...) toutes fonctions concernant directement les utilisateurs mais aussi aide aux acquisitions, au catalogage, au bulletinage, à la gestion (comptable, administrative, statistique ...).

Trop souvent encore cette introduction est vécue sans que soient suffisamment remises en cause l'organisation des tâches, les méthodes de travail, les relations professionnelles, comme si les nouvelles techniques pouvaient être «plaquées» sur des procédures conçues d'un bout à l'autre pour un travail «manuel». Une telle absence de réflexion critique constructive présente des inconvénients majeurs:

- alors que l'informatique et la télématique peuvent apporter des avantages certains en termes d'efficacité ou d'économie de moyens, ces objectifs, faute d'avoir été cernés, sont loin d'être correctement atteints;

- alors que ces mêmes techniques devraient permettre, par essence, une coopération extrêmement accrue de bibliothèques ou centres de documentation reliés en réseau, on assiste à la mise en place de systèmes «en vase clos», très peu aptes à dialoguer entre eux;

- alors qu'un emploi judicieux des nouvelles techniques doit conduire à une réduction, voire à une élimination des travaux les plus ingrats, en autorisant un véritable «enrichissement des tâches", si l'ensemble matériel-logiciel est bien adapté à son utilisation par les personnels des bibliothèques, il arrive qu'au contraire des contraintes inutiles s'introduisent, que des fatigues imprévues apparaissent, et parfois que des conflits de responsabilité se créent.

En conséquence, la mutation profonde de cette profession, alors qu'elle pourrait être vécue comme un enrichissement, est souvent ressentie avec inquiétude par les bibliothécaires et les documentalistes. Recensons quelques modifications essentielles intervenues dans les professions documentaires:

- cette profession était souvent choisie parce qu'elle offrait une possibilité de refuge pour des personnes préférant la fréquentation des livres aux rapports sociaux. Au fur et à mesure que se minimisent les tâches techniques, prises en charge par l'informatique, et que s'accroissent les tâches relationnelles (avec le public, avec les pairs, avec le secteur privé), tout ce qui faisait de cette profession un refuge disparaît. Cet élément déstabilisateur doit être pris en compte dans les actions de sensibilisation et de formation aux nouvelles technologies;

- la bibliothèque n'est plus un lieu de stockage des livres, mais un lieu d'accès à des services; le rôle du professionnel par rapport à l'usager se trouve totalement modifié, il devient un interface entre l'information et l'usager;

- il devient nécessaire que le bibliothécaire connaisse bien le domaine scientifique dont il s'occupe, afin de guider avec compétence les usagers dans leur recherche documentaire. La structuration des banques de données spécialisées accroît cette tendance;

- il n'est plus possible actuellement à un service de documentation de fonctionner en autarcie; un bibliothécaire ou un documentaliste ne peut plus maîtriser à lui seul la fonction documentaire:

- les bibliothèques fonctionnent en réseaux documentaires: la collaboration entre pairs devient indispensable;

- les nouvelles technologies imposent le recours à des personnes extérieures au monde de la documentation, tels que les informaticiens ou les sociétés de service.

Afin que la transition se fasse de manière heureuse, il convient de satisfaire à deux exigences: avoir le souci de l'ergonomie et adapter les formations et les modes de recrutement.

\section{- Le souci de l'ergonomie}

La conception d'un produit informatique implique certes la connaissance et le respect de normes ergonomiques générales; I'on devrait tenir compte en outre de la réalité des conditions de travail locales. À cette fin, une concertation est indispensable entre ceux qui vont installer un outil informatique et ceux qui vont l'utiliser: à défaut, l'utilisation d'un produit, même bien conçu sur le plan ergonomique, peut se faire de façon pervertie et dangereuse. Cette concertation devrait exister au moment de l'installation des matériels (où faut-il les installer? Quelle rotation de personnel est envisagée? ...) mais également en amont, dès la conception du produit: à ce stade, la collaboration des professionnels concernés permet de concevoir des outils parfaitement adaptés aux besoins. 
- L'adaptation des formations et des modes de recrutement

La formation initiale doit être repensée, pour s'adapter aux transformations intervenues dans les métiers de la documentation. La formation continue joue, quant à elle, un rôle essentiel pour la réussite de la transition: en effet, nombreux sont les professionnels actuellement en exercice que ni leur recrutement ni leur formation n'ont préparés aux tâches qui sont maintenant les leurs; en outre, la diversité des facultés d'adaptation est grande. La formation continue de ces personnels doit donc tenir compte de ces facteurs; ceci implique une concertation régulière entre formateurs et utilisateurs pour l'élaboration des stages, et un perpétuel réajustement en fonction des besoins des utilisateurs.

Je forme le voeu que ce colloque soit un lieu d'échanges fructueux sur les mesures à encou- rager ou à proposer:

- recommandations pour les chefs de projet et les équipes chargées de superviser et de développer les applications des nouvelles technologies;

- modes de concertation entre les responsables et les personnels concernés, avant et pendant la mise en oeuvre de ces applications, afin de tenir compte des aspects ergonomiques;

- approche ajustée des stages de formation mis en place au moment de l'introduction de ces applications, pour faire se rencontrer les profils et qualifications des personnels et les profils et spécificités des postes de travail;

- modification des formations initiales et continues, corrections à apporter aux critères de sélection pris en compte dans les concours de recrutement ...

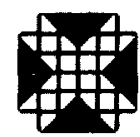

43. CONFERENCE ET CONGRES DE LA FID IFEDERATION INTERNAIIONALE LE DOCUMENATATEON MONTREAL. 14 AUL IE SEPTEMBRE 196

$43^{\text {rt }}$ FID CONFERENCE AND CONGRESS IMHTERNALIONAL FEDLRATON FOR GOCLMMENTATION MONTREAL, SEPTEMBER 14 TO 18,1985

\section{UNE INVITATION}

au

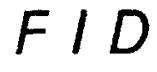

fédération internationale de documentation Fondée en septembre 1895

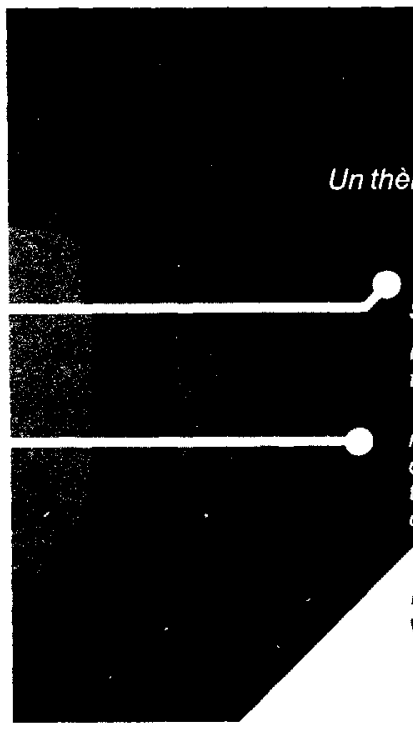

$43^{\circ}$ CONGRÈS DE LAFID

Montréal (Canada), 14-18 septembre 1986

\section{MFOERTOTHAN.}

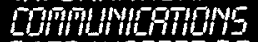

미

- ET TRAMSERT DE LATELHADLOBE

Sujets traités

instruments et système d'information télécommunications

nouvelles technologies de communication coordination et réseaux

transferts nationaux et internationaux de la technologie, etc.
Un programme complet

. 60 conférenciers de 21 pays

rencontres de spécialistes interprétation simultanée visites professionnelles activités sociales
Pour plus de renseignements, vous adresser au
COMITÉ LOCAL D'ORGANISATION

C.P. 1144 SUCCURSALE PLACE DESJARDINS, MONTREAL (QUEEBEC) CANADA H5B 1B3 TÉL.: (514) 288-8524 - TELEX 055-60944
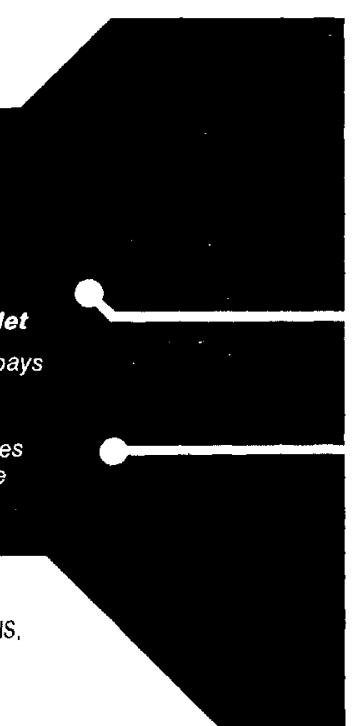